\section{Selection of legitimate dwarf coconut hybrid seedlings using DNA fingerprinting}

\author{
Alinne Oliveira Nunes Azevedo ${ }^{1 *}$, Carlos Diego de Oliveira \\ Azevedo ${ }^{1}$, Pedro Henrique Araújo Diniz Santos ${ }^{1}$, Helaine \\ Christine Cancela Ramos ${ }^{1}$, Marcela Santana Bastos Boechat ${ }^{1}$, \\ Fernanda Abreu Santana Arêdes ${ }^{1}$, Semíramis Rabelo Ramalho \\ Ramos $^{2}$, Luís Ângelo Mirizola ${ }^{3}$, Lalith Perera4, Wilson Meneses \\ Aragão ${ }^{5}$ and Messias Gonzaga Pereira ${ }^{1}$
}

\begin{abstract}
Due to the great economic importance of coconut palm in Brazil and the development of a coconut breeding program intended to produce intravarietal hybrids, the present work aimed to ease the production of hybrids with the same morphological heritage. DNA was extracted from leaf samples of 13 dwarf coconut populations from Brazil, and PCR amplification was performed using 21 previously selected microsatellites. Furthermore, this study selected nine microsatellite markers with the potential to identify Green Dwarf x Yellow Dwarf hybrids and 16 microsatellites with the potential to identify Red Dwarf $x$ Yellow Dwarf hybrids. In conclusion, SSR marker based on DNA Fingerprinting allowed the accurate identification of legitimate intravarietal hybrids since, for those crosses, the methodology of identification based on seedling color is not a viable alternative.
\end{abstract}

Keywords: Cocos nucifera L., intravarietal hybrid, microsatellite Markers.

\section{INTRODUCTION}

Coconut (Cocos nucifera L.) belongs to the single species of the genera Cocos and presents two varieties: Tall (var. typica) and Dwarf (var. nana). The Dwarf variety can be split into three color forms (subvarieties): Green Dwarf, Red Dwarf, and Yellow Dwarf. In addition, the red dwarf can be divided into two phenotypically distinct types: Malayan and Cameroon (Narayana and John 1949).

Coconut breeding studies aim to increase the albumen content, number of fruits per plant, sensorial characteristics, and resistance to pests, diseases, and drought (Liyanage 1967). One of the breeders' strategies to overcome such breeding constraints is the evaluation of intervarietal hybrids (Dwarf $x$ Tall). This method is used because these hybrids present heterosis for fruits production, earliness, resistance to pests and diseases, number of leaves, and stem girth (Santos et al. 1982).

Brazil is mostly interested in coconut water production, and therefore, the Green dwarf variety is the most suitable for cultivation owing to its high water yield and quality. The dwarf variety is the most used in coconut breeding
Crop Breeding and Applied Biotechnology 18: 409-416, 2018 Brazilian Society of Plant Breeding. Printed in Brazil http://dx.doi.org/10.1590/1984$70332018 \mathrm{v} 18 \mathrm{n} 4 \mathrm{a} 60$

\footnotetext{
*Corresponding author: E-mail: alinnenunes@live.com
}

Received: 12 March 2018 Accepted: 31 May 2018

${ }^{1}$ Universidade Estadual do Norte Fluminense Darcy Ribeiro, Laboratório de Melhoramento Genético Vegetal, 28.013-602, Campos dos Goytacazes, RJ, Brazil

${ }^{2}$ Embrapa Tabuleiros Costeiros, 49.025-040, Aracaju, SE, Brazil

${ }^{3}$ Comercial Regon Ltda, 32.628-070, Belo Horizonte, MG, Brazil

${ }^{4}$ Genetics \& Plant Breeding Division (CRISL), Lunuwila, Sri Lanka

${ }^{5}$ COHIBRA, Zona Rural Mirinduba, 62.540000, Amontada, CE, Brazil 
programs, as well as in the development of hybrids with the tall variety due to its earliness and yield in relation to other varieties (Nuce De Lamothe and Rognon 1977).

Therefore, the Plant Breeding Department of Universidade Estadual do Norte Fluminense Darcy Ribeiro has started a study aiming to explore heterosis of intravarietal hybrids of dwarf coconut and identify hybrids resistant to diseases and with the highest yield and the best water quality.

However, heterosis exploitation can be damaged because dwarf coconut tends to be predominantly autogamous, with a low proportion of allogamy (depending on the subvariety), resulting in low reliability of a successful hybridization.

Thus, hybrid seedlings need to be selected with a greater rigor during the genetic purity verification. For instance, morphological parameters (e.g., petiole color and growth vigor) can suffer environmental variation, which can result in the undesirable selection of cross-pollinated siblings and rejection of good hybrids. The problem can be doubled if the selection is related to planting commercial seeds, in which the selection of impure types can result in a waste of time and resources and the loss of desirable genetic purity (Rajeshet al. 2013). Therefore, a strategy must be developed to help breeders identify pure seedlings of intravarietal hybrids (Dwarf x Dwarf) with greater reliability.

In fact, no reliable method has been reported for the confirmation of coconut hybrids identity. Due to the long juvenile period and the perennial nature of coconut, the lack of precise hybrid identification generates great problems during seed production and coconut cultivation.

Molecular markers have been applied to the identification, plant or variety registry, and purity control of hybrid seeds, promoting authenticity with reliability, high precision, and low cost (Hoffmann and Barroso 2006). Moreover, molecular markers are less influenced by the environment than morphological markers and can be detected at any plant growth stage (Guimarães and Moreira 2005). Accordingly, the use of molecular markers tends to be a better alternative to prove the legitimacy of intravarietal hybrids.

DNA Fingerprinting is a molecular technique that has been used to describe the molecular pattern of a genotype and can be obtained by several types of molecular markers. Codominant markers (microsatellites, SNP, CAPS, and SCAR) are the most recommended for DNA Fingerprint, mainly regarding cultivar protection.

Rajesh et al. (2013) identified a marker related to plant height using RAPD. Perera (2010) identified two pairs of microsatellite primers, which are more specific than the SCAR primers from the study of Rajesh et al. (2013). Such microsatellites could successfully distinguish Sri Lanka Tall (SLT) from Sri Lanka Green Dwarf (SLGD) and Sri Lanka Yellow Dwarf (SLYD). Preethi et al. (2016) used EST-SSR markers to test the genetic purity of the progeny obtained from hybrids TALL X DWARF from ICAR-CPCRI Farm (Kasaragod, Kerala, India). No studies have evidenced the identification of intravarietal hybrids (Dwarf X Dwarf) by molecular markers.

Therefore, the present study aimed to obtain DNA fingerprints for 13 dwarf coconut populations from Brazil using microsatellite markers. These populations are also being used by the coconut breeding program of Universidade Estadual do Norte Fluminense Darcy Ribeiro (UENF). Moreover, this study intended to verify the legitimacy of hybrid seeds of dwarf $x$ dwarf crossings using the detected markers, aiming to promote intravarietal hybrids production.

\section{MATERIAL AND METHODS}

\section{DNA fingerprinting identification}

The methodology was developed at two stages, microsatellite markers screening and specific markers identification, followed by their validation with hybrid samples. First, bulks of samples representing 13 dwarf coconut populations from different growing regions of Brazil were used to screen SSR primers. These populations are parents of intravarietal hybrids from an assay involving a Top Cross design that is being developed by the coconut breeding program of UENF.

The 13 populations under study are: Brazilian Green Dwarf - Jiqui (BGDJ), Brazilian Green Dwarf - Paraipaba (BGDPRPB), Brazilian Green Dwarf - Bahia (BGD-BA), Brazilian Green Dwarf - Souza 1 (BGD-SZ 1), Brazilian Green Dwarf - Souza 2 (BGD-SZ 2), Brazilian Green Dwarf - Pará 1 (BGD-PA 1), Brazilian Green Dwarf - Pará 2 (BGD-PA 2), Brazilian 
Green Dwarf - Trairi (BGD-TR), Malayan Red Dwarf (MRD), Malayan Yellow Dwarf (MYD), Brazilian Red Dwarf Gramame (BRDG), Cameroon Red Dwarf (CRD), and Brazilian Yellow Dwarf - Gramame (BYDG).

\section{Hybrids validation}

Two hundred samples of the hybrid BGDJ x BYDG; 25 samples of the hybrid BGDJ x CRD; 25 samples of the hybrid BGDJ x BRDG; and 25 samples of the hybrid BGDJ x MRD were used for hybrid validations tests. Samples of the hybrids Green Dwarf x Yellow Dwarf were randomly chosen at a nursery because in such type of crossing when Green Dwarf is the female parent, phenotypic identification of hybrids is difficult. However, in the case of the hybrids green dwarf $x$ red dwarf, samples were selected based on morphological markers, using 15 legitimate samples (hybrids) and ten nonlegitimate samples (selfing) for markers validation in the laboratory.

\section{Genomic DNA isolation}

DNA was extracted using the DNeasy Plant Mini Kit (QIAGEN). For the identification of potential markers for fingerprinting, each one of the 13 populations formed a bulk composed of leaf samples from each genotype of the population. For the validation of the selected potential markers, each sample had its DNA extracted individually. DNA was quantified by spectrophotometry (NanoDrop 2000C - Thermo Scientific), and concentrations were standardized at $5 \mathrm{ng} \mu \mathrm{L}^{-1}$.

\section{Microsatellite analysis}

DNA amplification reactions were performed according to the PCR protocol proposed by Baudouin (2009), at a final volume of $20 \mu \mathrm{l}$ and an annealing temperature of $51 \stackrel{\circ}{\circ}$. Twenty-one pairs of microsatellite primers were used to identify specific markers for the populations under study (Table 1). Afterward, markers were validated in the hybrids. PCR products underwent a Capillary system of electrophoresis (Fragment Analyzer - AATI), in which the amplified fragments at a range from 35 to $500 \mathrm{bp}$ are separated with a resolution of about $2 \mathrm{bp}$.

Table 1. List of sequences of SSR primers for coconut used in this work

\begin{tabular}{|c|c|c|}
\hline Primers & $\begin{array}{c}\text { Forward primer } \\
\left(5^{\prime}-3^{\prime}\right)\end{array}$ & $\begin{array}{c}\text { Reverse primer } \\
\left(5^{\prime}-3^{\prime}\right)\end{array}$ \\
\hline CnCir $A 9^{1}$ & AATGTTTGTGTCTTTGTGCGTGTGT & ТССТТАТТTТТСТТССССТТССТСА \\
\hline CnCir G11 ${ }^{1}$ & AATATCTCCAAAAATCATCGAAAG & TCATCCСАСАСССТССТСТ \\
\hline CnCir $\mathrm{H}_{11}{ }^{1}$ & TCATTCAGAGGACAAAAGTT & TAAAAATTCATAAAGGTAAAA \\
\hline $\mathrm{CAC}^{3}{ }^{3}$ & CCССTATGCATCAAAACAAG & CTCAGTGTCCGTCTTTGTCC \\
\hline CAC $46^{4}$ & GATGGTTGGATATCATTCTTG & TTGACCTATCAAATGTGCC \\
\hline CAC 55 & CTATGCATCAAAACAAGGAG & CAAATCTAGGTAGCTTGAGGT \\
\hline $\mathrm{CAC}^{2} 5^{3}$ & GAAAAGGATGTAATAAGCTGG & TTTGTCCCCAAATATAGGTAG \\
\hline CAC6 $7^{4}$ & GGAGAAACGGTATACCAGAG & СCTCATTTAGATGCCCTATC \\
\hline $\mathrm{CAC} 69^{4}$ & TATAAATGGGTAGCCCTGAG & TGAATAGGTTGGTGAATGTG \\
\hline CAC83 & AATTGAAACCAACCCAACAA & TTGCCATGTTTTACTTGTAGC \\
\hline $\mathrm{CNZO1}^{1}$ & ATGATGATCTCTGGTTAGGCT & AAATGAGGGTTTGGAAGGATT \\
\hline $\mathrm{CNZO6}^{2}$ & ATACTCATCATCATACGACGC & CTCCCACAAAATCATGTTATT \\
\hline $\mathrm{CNZ10}{ }^{2}$ & CCTATTGCACCTAAGCAATTA & AATGATTTTCGAAGAGAGGTC \\
\hline CNZ292 2 & TAAATGGGTAAGTGTTTGTGC & CTGTCCTATTTCCCTTTCATT \\
\hline $\mathrm{CNZ46}^{2}$ & TTGGTTAGTATAGCCATGCAT & AACCATTTGTAGTATACCCCC \\
\hline
\end{tabular}

${ }^{1}$ Baudouin (2009); ${ }^{2}$ Rivera (1999); ${ }^{3}$ Perera et al. (2000); ${ }^{4}$ Mauro-Herrera et al. (2010). 


\section{Data analysis}

Electrophoresis data were tabulated, and genetic distances between populations were calculated using the software GenAlEx (Peakall and Smouse 2012). The genetic distance matrix was used to generate the clustering of genotypes by the software MEGA version 5 (Tamura et al. 2011). The clustering was performed by the UPGMA method (Unweighted Pair Group Method with Arithmetic Means). The descriptive analysis was performed by the GenAlEx (Peakall and Smouse 2012) and PowerMarker (Liu and Muse 2005) software. These analyses provided the estimate of genetic parameters and allowed a more accurate inference about the populations under study.

\section{RESULTS AND DISCUSSION}

The 21 loci under evaluation totaled 56 alleles, of which 36.5 are effective alleles with a mean of two alleles per locus (Table 2). PIC values varied from 0.13 to 0.56 , with a mean of 0.33 . The higher PIC value was obtained by the primer CAC55, and the lower values were obtained by the primers CAC 46, CAC 67, CAC 82, and CNZ 10. The loci CAC 46, CAC 67, CAC 82, and CNZ 10 presented a higher probability of identity $(I=0.75)$, which is justified by their low polymorphism. The combined probability of exclusion presented low values; however, the primer CAC 55 can be pointed as the most viable for distinguishing these genotypes. Primers were effectively discriminatory for the identification of each dwarf coconut subvariety.

Some studies have been successful in identifying DNA fingerprints that mainly differentiate the varieties dwarf from tall, aiming to apply them in the verification of the authenticity of intervarietal hybrids (Perera 2010, Rajesh et al. 2012, Rajesh et al. 2013). However, identifying specific markers for the different forms of dwarf coconut is also fundamental. This fact is explained by the possibility of working with intravarietal hybrids and because the phenotypic marker does not always work at $100 \%$ reliability.

Coconut hybrid seedlings can be identified in the nursery by two methods: stem color and molecular markers. The plant color is an example of a morphological marker that can help identify hybrids. According to Bourdeix (1988), the

Table 2. Locus, Allele Size, Major Allele Frequency, Number of alleles, Number of effective alleles per locus, Polymorphic Information Content (PIC), Probability of Identity (I), and Exclusion Probability (Q)

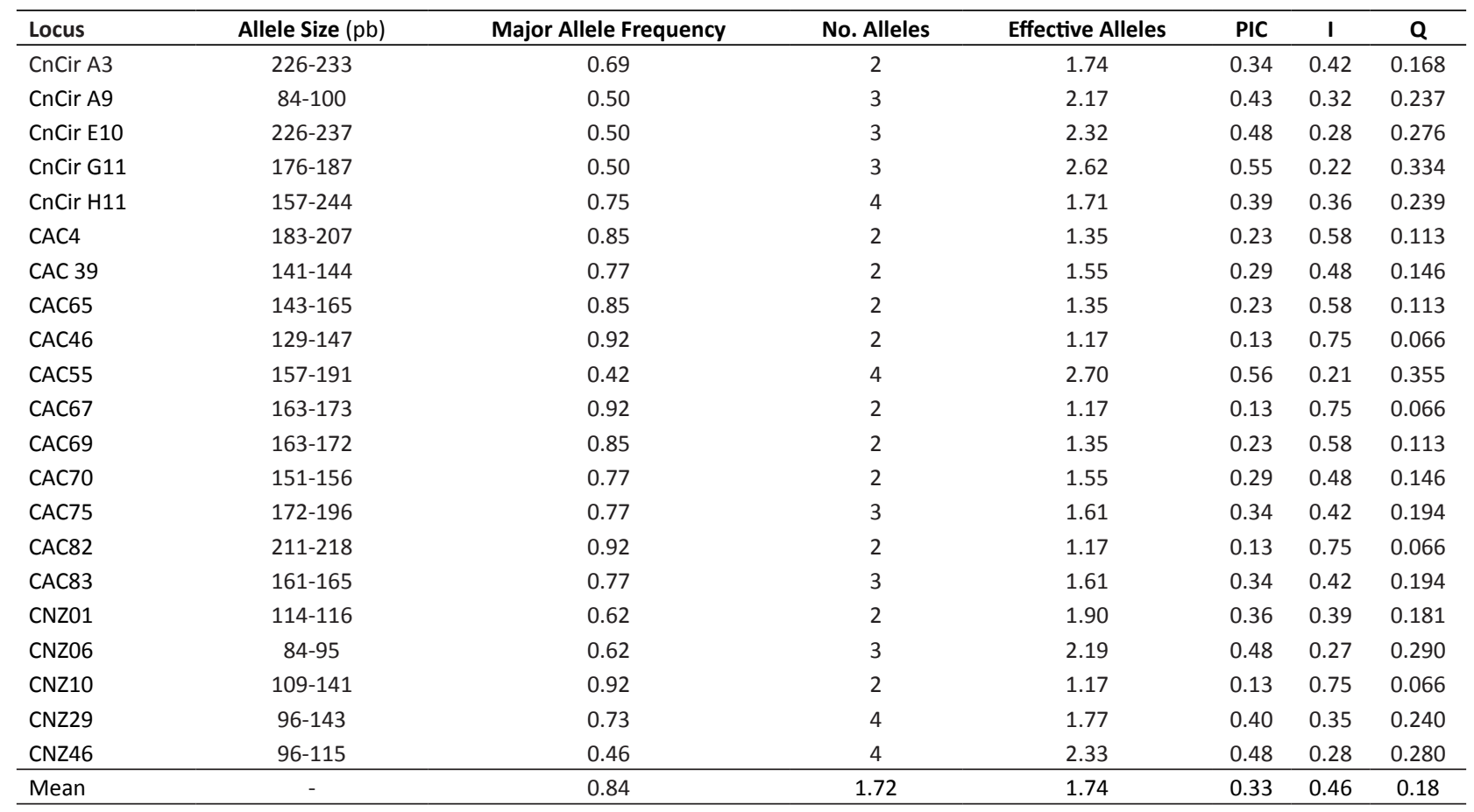


Table 3. Morphological and Molecular Markers for the identification of intravarietal hybrid combinations

\begin{tabular}{lccc} 
Female Parent & Male Parent & & Identification \\
Green Dwarf & Red Dwarf & Phenotypic & Brown \\
Red Dwarf & Green Dwarf & Phenotypic & Brown \\
Green Dwarf & Yellow Dwarf & Molecular & CnCir A3, CnCir G11, CAC4, CAC 55, CAC65, CAC69, CAC83, CNZ01, CNZ06 \\
Yellow Dwarf & Green Dwarf & Phenotypic & Green \\
Red Dwarf & Yellow Dwarf & Molecular & CnCir A9, CnCir E10, CAC4, CAC 39, CAC 46, CAC55 CAC65, CAC67, CAC69, \\
Yellow Dwarf & Red Dwarf & Phenotypic & CAC75, CAC82, CAC83, CNZ06, CNZ10, CNZ29, CNZ46 \\
\hline
\end{tabular}

color of coconut fruit results from the expression of two independent genes, coded as " $R, r, G$, $g$ ", in which: the double recessive genotype codifies to yellow phenotype; genotypes whose allele " $r$ " dominates the allele " $g$ " codify to red phenotype; genotypes whose allele " $g$ " dominates the allele " $r$ " codify to green phenotype; and other combinations produce brown phenotype.

Aragão et al. (1999) tested all possible combinations of all genotypes and phenotypes generated in relation to those two genes that control color heritage, including reciprocals. They concluded that, depending on the genotype chosen as the female parent, the identification of the hybrid by a phenotypic marker is not viable.

Table 3 shows that the identification by morphological markers is effective only for the crosses between Green Dwarf x Red Dwarf and its reciprocal. For crosses between Green Dwarf x Yellow Dwarf and Red Dwarf x Yellow Dwarf, the morphological marker is only effective when yellow dwarf is used as the female parent. Thus, the markers selected in the present study can be an excellent tool to evaluate the legitimacy of these hybrid combinations, mainly in those cases in which the morphological markers are incapable of detecting the legitimacy.

Table 4 shows the identified alleles for each population, as well as each primer pair that can be used to identify crosses obtained from a Top Cross. Since all populations were crossed with BGDJ, only one population needs to present a different allele from the tester (BGDJ) so that the primer can be satisfactory for seeds verification from this crossing. In the case of the crossing BGDJ X CRD, primers CnCir A3, CnCir A9, CnCirE10, CnCir G11, CAC 39, CAC 46, CAC 67, CAC

Table 4. Primers that presented specific alleles for each population of dwarf coconut

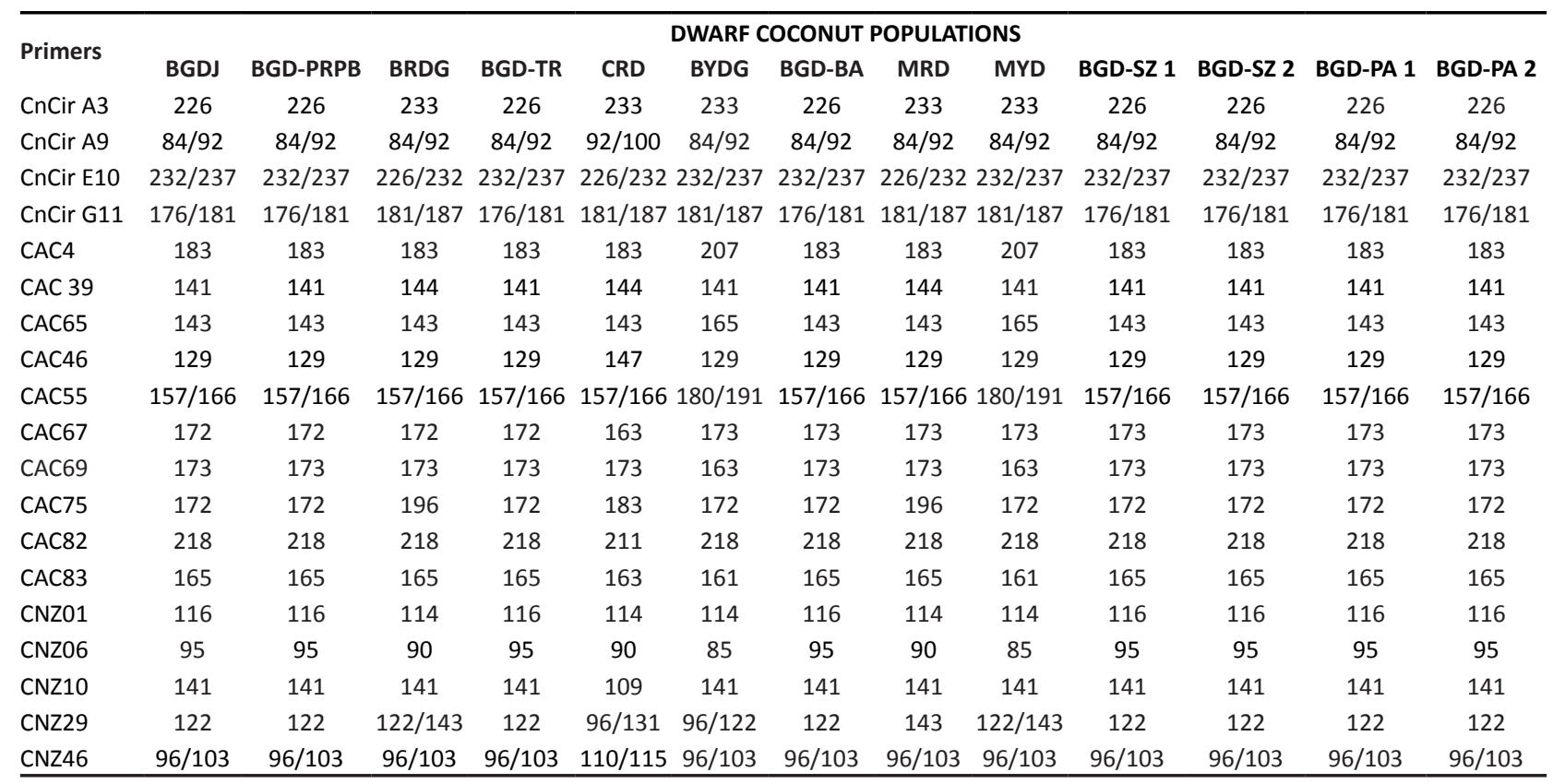




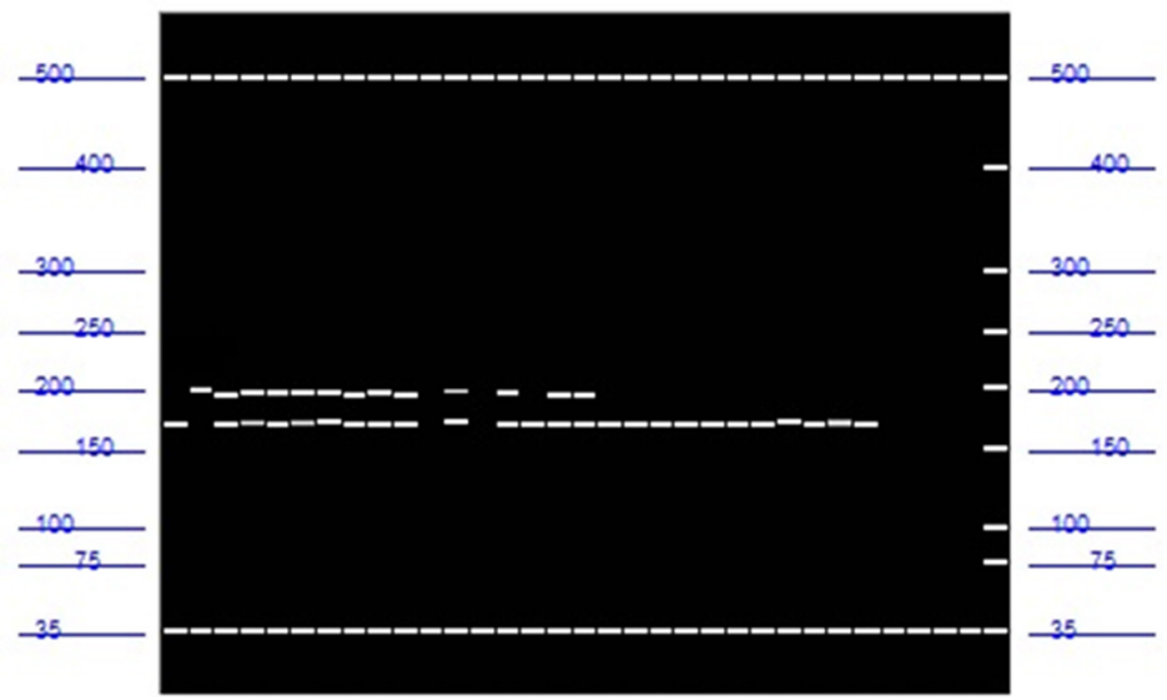

Figure 1. Capillary electrophoresis gel obtained by the software ProSize 2.0 showing parents (BGDJ X BRDG), hybrids seedlings, and illegitimate seedlings, utilizing the primer CAC 75.

75, CAC 82, CAC 83, CNZ 01, CNZ 06, CNZ 10, CNZ 29 and CNZ 46 are applicable to distinguish this seedling in the nursery because both populations show contrasting alleles for the same locus.

The detection of these primers will allow higher reliability for the verification of seeds genetic purity, which will avoid wasting time and resources and losing genetic purity in experimental or commercial fields. Molecular genotyping can be performed during nursery time, and only seedlings identified as legitimate hybrids can be taken to the field. Furthermore, considering that each coconut tree occupies an area of about $42 \mathrm{~m}^{2}$, planting a non-hybrid seedling could lead to losses for the farmer.

\section{Hybrids validation}

\section{Green Dwarf x Red Dwarf}

Green Dwarf x Red Dwarf hybrids can be previously identified by a morphological marker (stem color) when they are in the nursery. To validate the markers, two bulk parents, 15 samples of legitimate hybrids, and ten illegitimate hybrids (selfing) were used, according to the morphological marker.

Table 3 shows that all illegitimate seedlings presented green stem. This result is because they are derived from selfing, and the female parent used in the assay was BGDJ. Hybrid seedlings presented brown stem. For the crossing BGDJ X BRDG, only the primer CAC 75 (Figure 1) obtained the same segregation pattern of the morphological markers. In other words, when the stem was brown, the genotype presented bands of both parents, and when the stem was green, the genotype presented bands of only the female parent, indicating that the seed was originated by selfing. The same event was detected for the crosses BGD-JIQUI X GRD and BGD-JIQUI X CRD using the primers CnCir A3, CnCir G11, CAC 39, CNZ 01, and CNZ 06.

\section{Green Dwarf x Yellow Dwarf}

Hybrids Green Dwarf x Yellow Dwarf were validated at two stages. At first, nine primers (previously identified) were tested in all individuals of the populations Brazilian Yellow Dwarf of Gramame and Brazilian Green Dwarf of Jiqui. The test intended to verify which marker was fixed in the populations to obtain greater safety. This procedure was used because the identification by the morphological marker is not possible for this type of hybrid.

Considering the electrophoretic profile of bulks, the primers cited in table 3 would be reliable for hybrids identification. 
The primer $\mathrm{CnCir}$ A3 presented the major alleles fixation for the genotyping of individual samples and was selected for the hybrids identification in the nursery. This primer worked satisfactorily, clearly distinguishing hybrids from selfed or illegitimate seeds. The genotyping of the samples detected $87 \%$ of legitimate hybrids, which were recommended for safe and precise planting.

During the validation of the identified markers, low alleles fixation was observed in each locus, which can be a consequence of the methodology of bulking samples at the first stage. Since the bulks were composed of leaf samples that were smashed together, this procedure might have provoked low representativity of some alleles, masking the analysis of the screening.

Otherwise, such a problem can be solved when the parent genotypes are known before hybridization. By a previous genetic analysis of parents, only those that present the allele of interest for crossing can be selected, and thus all primers identified at the screening can be successfully used for hybrid seeds identification.

Heretofore, specific markers to differentiate intravarietal crossings, such as Green Dwarf x Green Dwarf, have not been identified yet. Nevertheless, the lack of variability detected between the green dwarf populations can be justified by their narrow genetic relationship, as previously described by some authors. Perera et al. (2000) stated that dwarf coconut possibly evolved during a long period of domestication from a small population of tall coconut, which indicates that such event can be the reason for the low levels of genetic diversity in dwarf coconut. This result implies a smaller genetic distance between Brazilian dwarf coconut accessions and a higher level of genetic relationship.

\section{CONCLUSION}

The DNA Fingerprinting technique allowed the accurate identification of legitimate seedlings originated by intravarietal dwarf $x$ dwarf hybrids, mainly hybrids originated by crosses between Green Dwarf x Yellow Dwarf and Yellow Dwarf $x$ Red Dwarf. It is important to emphasize that for such crossings when the female parent is the green dwarf, the methodology of identification based on the color of the seedling is not a $100 \%$-viable alternative.

\section{ACKNOWLEDGMENTS}

The authors are grateful to the Brazilian institutions Universidade Estadual do Norte Fluminense Darcy Ribeiro, FAPERJ, EMBRAPA, COHIBRA, and REGON, for enabling the accomplishment of this study.

\section{REFERENCES}

Aragão WM, Câmara TM and Ribeiro FE (1999) Marcadores morfológicos em coqueiros (Cocos nucifera L.). EMBRAPA- CPATC, Aracaju-SE, 20p. (Documentos 7).

Baudouin L (2009) Consolidate microsatellite data on coconut diversity: Appendices. CIRAD, Montpellier, 42p.

Bourdeix R (1988) Genetic determinism in dwarf coconut germ colour. Oleagineux 43: 371-374.

Guimarães CT and Moreira MA (2005) Genética molecular aplicada ao melhoramento de plantas. In Borem A (ed) Melhoramento de espécies cultivadas. $2^{\text {nd }}$ edn, UFV, Viçosa, 969p.

Hoffmann LV and Barroso PAV (2006) Marcadores moleculares como ferramentas para estudos de genética de plantas. Embrapa Algodão, Brasília, 35p. (Documentos 147).

Liyanage DV (1967) Identification of genotypes of coconut palms suitable for breeding. Experimental Agriculture 3: 205-210.

Liu K and Muse SP (2005) PowerMarker: an integrated analysis environment for genetic marker analysis. Bioinformatics Applications Note 21: 2128-2129.

Mauro-Herrera M, Meerow A W, Perera L, Russell J and Schnell R J (2010) Ambiguous genetic relationships among coconut (Cocos nucifera L.) cultivars: the effects of out-crossing, sample source and size, and method of analysis. Genetic Resources and Crop Evolution 57: 203-217.

Narayana GV and John CM (1949) Varieties and forms of the coconut. Madras Agricultural Journal 36: 349-366.

Nuce De Lamothe $M$ and Rognon $F$ (1977) Les cocotiers nains à Port - Bouêt Nain Jaune Ghana, Nain Rouge Malais, Nain vert Guiné Equatoriale, Nain Rouge Cameroun. Oléagineux 32: 367-373.

Peakall R and Smouse PE (2012) GenAlEx 6.5: genetic analysis in Excel. Population genetic software for teaching and research - an update. Bioinformatics 28: 2537-2539.

Perera L, Russell JR, Provan J and Powell W (2000) Use of microsatellite DNA markers to investigate the level of genetic diversity and population genetic structure of coconut (Cocos nucifera L.). Genome 43: 15-21. 
Perera L (2010) Hybrid testing and variety identification of coconut (Cocos nucifera L.) in Sri Lanka using microsatellite markers. Cord 26: 39-43.

Preethi P, Rajesh MK, Rahul CU, Jerard BA, Samsudeen K, Thomas RJ and Karun A (2016) Identification and utilization of informative EST-SSR markers for genetic purity testing of coconut hybrids. Journal of Plantation Crops 44: 77-84.

Rajesh MK, Thomas RJ, Rijith J, Shareefa M and Jacob PM (2012) Genetic purity assessment of D x T hybrids in coconut with SSR markers. Indian Jornal Genetic 72: 472-474.

Rajesh MK, Jerard BA, Preethi P, Thomas RJ and Fayas TP (2013) Development of a RAPD-derived SCAR marker associated with tall- type palm trait in coconut. Scientia Horticulturae 150: 312-316.

Rivera R, Edwards KJ, Barker JH, Arnold GM, Ayad G, Hodgkin T and Karp A (1999) Isolation and characterization of polymorphic microsatellites in Cocosnucifera L. Genome 42: 668-675.

Santos GA, Carpio CB, Ilagan MC, Cano SB and Delacruz BV (1982) Flowering and early performance of four IRHO coconuts hybrids in the Philippines. Oléagineux 37: 571-580.

Tamura K, Peterson D, Peterson N, Stecher G, Masatoshi N and Kumar $S$ (2011) MEGA5: Molecular evolutionary genetics analysis using maximum likelihood, evolutionary distance, and maximum parsimony methods. Molecular Biology Evolution 28: 2731-2739. 